\title{
CASE REPORT \\ A RARE PRESENTATION OF ATRIAL FIBRILLATION AND THROMBOTIC MICROANGIOPATHY, (THROMBOTIC THROMBOCYTOPENIC PURPURA - TTP) CAUSED BY ALCOHOL AND PRODUCTS OF CANNABIS (MARIJUANA)
}

\author{
Samar Iltaf', Saira Awan', Saira Abbas', Salma Salman', Shariq Mirza1 \\ ${ }^{1}$ Dow University of Health Sciences, Karachi, Pakistan
}

Cannabis used as both for medicine as well as recreational purposes. Extensive use is associated with many documented central nervous system and hepatic manifestation. How-ever only few cases are reported in literature with cardiac and hematological manifestation. Our case report is one of its kinds where our patient presents with thrombotic thrombocytopenic purpura and atrial fibrillation and he required extensive treatment including plasmapharesis.

Keywords: valvular aortic stenosis, valvular pulmonary stenosis, semilunar valves, balloon valvoplasty

Citation: Iltaf S, Awan S, Abbas S, Salman S, Mirza S. A Rare Presentation of Atrial Fibrillation and Thrombotic Microangiopathy, (Thrombotic Thrombocytopenic Purpura - TTP) Caused By Alcohol and Products of Cannabis (Marijuana). Pak Heart J. 2021;54(04):373-376. DOI: https://doi.org/10.47144/phj.v54i4.2082

\section{INTRODUCTION}

Thrombotic Thrombocytopenic Purpura (TTP) is a rare form of a blood disorder known as microangiopathic hemolytic anemia (MAHA) characterized by the consumption of platelets and shearing of red blood cells (RBCs). It is due to a decrease in the levels of the enzyme ADAMTS13 (a disintegrin and metalloproteinase 13), ${ }^{1}$ usually acquired in the adult female population via the production of autoantibodies. TTP may be inherited or acquired.

The triggering factors include pregnancy, cancer, systemic lupus erythematosus (SLE), HIV (human immunodeficiency virus), and medications such as Quinine, Clopidogrel, Cyclosporin-A, Chemotherapy drugs, and rarely certain specific medical procedures such as bone marrow or stem cell transplantation. Atrial fibrillation is a very common arrhythmia and there are several causes, one of the rare etiology is alcohol which is reported in 13-36 percent of the population. $^{2}$

The frequency of cannabis and alcohol consumption in the younger population is rapidly rising on a widespread scale throughout the globe. In Pakistan, the consumption of cannabis and alcohol has sharply increased in the recent decade, as appose to previous years, as such, there has been a local influx of reported alcohol and cannabis-related patient cases. ${ }^{3}$

We are reporting this case after taking verbal and written consent from the patient and department as well. In this case report our patient presented with an uncommon complication of alcohol and cannabis intake, exhibiting advanced symptoms of a rare hematological disorder identified as thrombotic microangiopathy and atrial fibrillation.

\section{CASE REPORT}

A 36-year-old male driver by profession, married, addicted to alcohol, hashish, and marijuana, received through emergency in the intensive care unit, with complaints of high-grade fever for one day followed by episodes of generalized tonic-clonic fits and altered level of consciousness. On general physical examination, a young male of average height and built lying on bed intubated and on Assist/control mode of a ventilator, his blood pressure was $145 / 79 \mathrm{mmhg}$, pulse $140 \mathrm{~b} / \mathrm{m}$ regularly irregular, respiratory rate was $18 \mathrm{~b} / \mathrm{min}$, temperature $102^{\circ} \mathrm{F}$, he was intubated and on Assisted/Control mode of a ventilator, $\mathrm{FiO} 2$ 90\%, frequency $20 \mathrm{~b} / \mathrm{m}$, tidal volume $450 \mathrm{ml}$, PEEP 5.0, further general physical examination showed no abnormalities (in particular no splinter hemorrhages, no lymphadenopathy), rest of the systemic examination was unremarkable other than neurological examination.

Neurological examination at the time of admittance showed a young male not oriented to time place and person, Glasgow coma scale (GCS) 3/10T, signs of meningeal irritation were absent, pupils dilated and reactive to light, fundus examination showed blurred disc margins, rest of the cranial nerves not were assessed, there was no facial asymmetry, sensory and cerebellar examination, as well as gait examination not assessed. Motor examination bulk was normal, the tone was increased in all four limbs, power was $2 / 5$, reflexes were intact, Babinski reflex was positive bilaterally. 
Routine blood examination showed hemoglobin level of $8.5 \mathrm{mg} / \mathrm{dl}$, MCV $82 \mathrm{fl}$, WBC $9.0 \times 109 / \mathrm{L}$, Platelet count 40,000, fragmented RBC, schistocytes, anisocytosis were detected on peripheral film, retic count $3.0 \%$, direct coombs test was negative, $\mathrm{LDH}$ 1100 , serum urea 194.7 , creatinine 8.15 , sodium $138 \mathrm{mEq}$, potassium $3.9 \mathrm{mEq}, \mathrm{HCO} 3$ 23.2, ADAMTS13 was not performed, serum total bilirubin $2.22 \mathrm{mg} / \mathrm{dl}$, conjugated bilirubin $0.7 \mathrm{mg} / \mathrm{dl}$, unconjugated bilirubin $1.52 \mathrm{mg} / \mathrm{dl}$, SGPT $417 \mathrm{u} / \mathrm{l}$, SGOT $57 \mathrm{u} / \mathrm{l}$, ALK phosphatase $257 \mathrm{u} / \mathrm{l}$, serum amylase 40u/l.

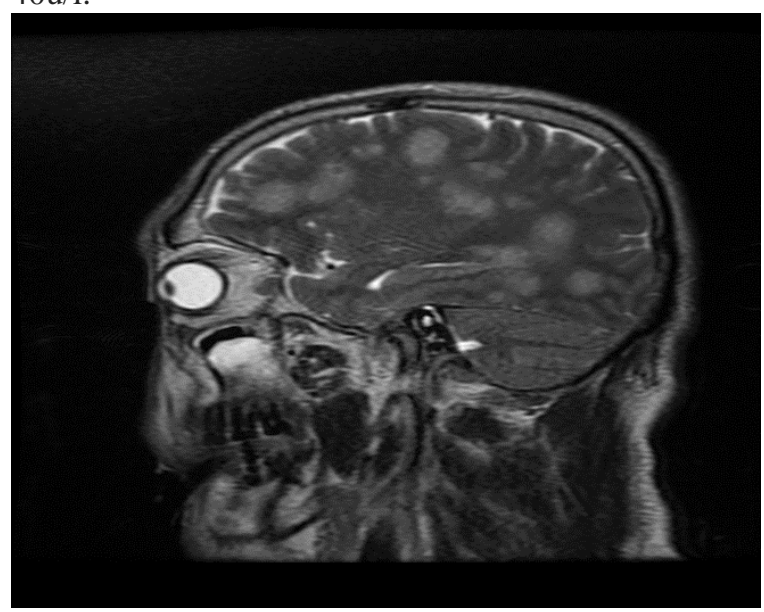

Figure 1: MRI brain T2-weighted coronal section

MRI brain T2-weighted image, showing multiple hyper-intensity areas on frontal, parietal and occipital regions.

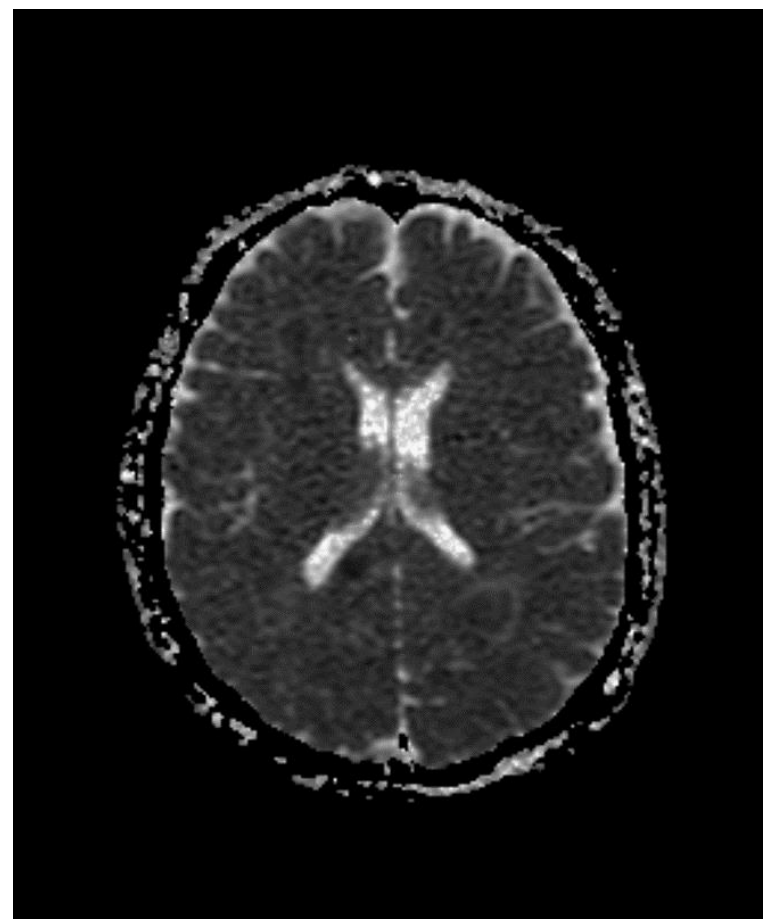

Figure 2: MRI Brain apparent diffusion coefficient image

Bilateral, hypo-intensity areas on ADC map of MRI brain.

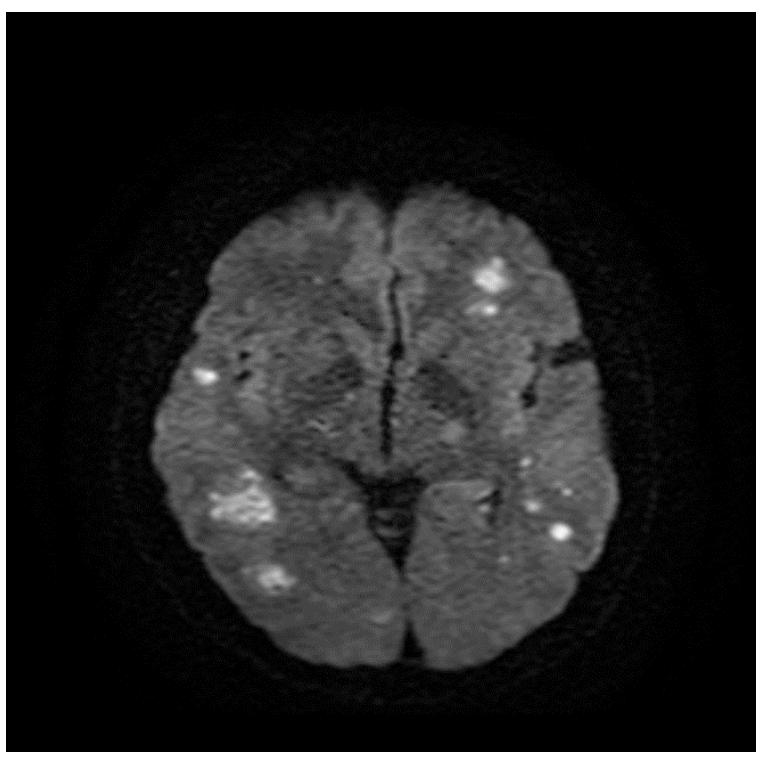

Figure 3: MRI brain diffusion weighted image bilateral, multiple, hyper-intensity areas on the diffusion-weighted image (diffusion restriction).

HIV, HBsAg, anti HCV non-reactive, C-reactive protein $90.79 \mathrm{mg} / \mathrm{l}$, D-dimers $80.00 \mathrm{mg} / \mathrm{dl}$, ESR 18, Troponin-I 817pg/ml, CPK 3670, PT/INR 12.4/1.16, urine detail report hemoglobinuria, protein-urea $0.4(1+)$, blood and urine culture and sensitivity show no growth, CSF studies showed appearance clear, glucose $85 \mathrm{mg} / \mathrm{dl}$, protein 42 , TLC $2.0 / \mathrm{mm} 3, \mathrm{C} / \mathrm{S}$ no growth, autoimmune encephalitis AMPA, CASPR, LG11, NMDA not detected in CSF, ANA \& ENA profile not detectable, ultrasound abdomen unremarkable, ECG showed atrial fibrillation echocardiography Figure 4. Ejection fraction 65\%, left ventricle function was normal, TSH normal. EEG was abnormal with generalized slowing due to encephalopathy. 


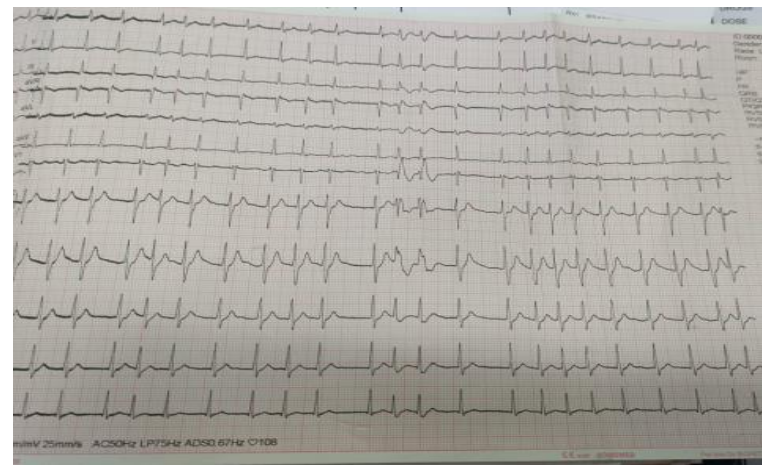

Figure 4: ECG showing atrial fibrillation,

MRI brain with gadolinium contrast images, T1, T1 contrast Diffusion-weighted image DWI and apparent diffusion coefficient ADC images, show bilateral, multifocal, subcortical and deep, supra-tentorial and infra-tentorial lesions, which are hypo-intense signals on T1, hyper-intense on T2 with no post-contrast enhancement, low on ADC map and show diffusion restriction on DWI [Figure 1-4]. Almost all of them contain internal susceptibility representing hemorrhage. Findings are suggestive of hemorrhagic infarct/thrombotic microangiopathy.

During admission, multiple rounds of dialysis were performed. Urgent plasmapheresis was performed, 7 consecutive cycles of plasmapheresis with fresh frozen plasma FFP performed, followed by alternateday Plasmapheresis. During admission, the patient has agitation and aggressive behavior upon which psychiatric consult was called. Urine toxicology indicated the patient had been consuming alcohol. For sedation, IV propofol and midazolam was used. Meanwhile, the blood picture was getting worse over time, $\mathrm{HB}$ and platelets were getting low and the patient went in disseminated intravascular coagulation for that multiple transfusion of RBC and FFP performed.

This showed the classic pentad of TTP upon which the patient was started on plasmapheresis. The patient's condition started to get better. His renal function, LDH, platelet count improved. The patient was kept on plasmapheresis for 15 days and eventually was stepped down from ICU and discharged later on

\section{DISCUSSION}

We are reporting a rare case of a young age patient with atrial fibrillation and TTP who was a marijuana and alcohol addict. TTP shows a classic pentad of Thrombotic microangiopathy.

Thrombotic microangiopathy (TMA) is a rare hematological disorder that is characterized by thrombosis in small blood vessels, low platelet count, activation of the coagulation cascade, the disease is characterized by the following pentad:

Neurovascular abnormalities, Microangiopathic hemolytic anemia, Thrombocytopenic purpura, Pyrexia, Renal vessel disease. ${ }^{4}$

The actual etiology of TTP/TMA is still unknown. ${ }^{2}$ Most cases of TTP are associated with autoantibodies against the protease ADAMTS13 activity. Measuring ADAMTS13 levels may help in the diagnosis of TTP $^{5,6}$ accumulation of large multimers of von Willebrand factor (vWF) in the plasma due to the lack of plasma protease that is responsible for the breakdown of these ultra-large vWF multimers.

TTP is also associated with drugs such as alkylating agents, Cisplatin, Mitomycin, Bleomycin, Cyclosporine, Ticlopidine, Quinine, OCP, and HRT. Heavy binge alcohol and cannabinoids are one of the major precipitating factors of Stroke, MI, atrial fibrillation, and TTP by thromboxane-related platelet aggregation, alcohol can cause depletion of $\mathrm{Mg} 2+$, which further aggravates vasospasm. ${ }^{7}$

Previously only one case was reported of an adult male who presented with acute kidney injury, after taking synthetic products of cannabinoids, he was found to have thrombotic microangiopathy (TMA) organ injury, proven by biopsy. ${ }^{8}$ TTP is a medical emergency and management should be started as soon as possible. ${ }^{9}$ Plasmapheresis with (FFP) fresh frozen plasma is the treatment of choice, if plasma exchange is not available, plasma infusions can also be performed. ${ }^{9}$

In conclusion, we are presenting a very rare case with severe hematological and cardiac manifestations of binge alcohol drinking and cannabinoids synthetic products, atrial fibrillation, and thrombotic thrombocytopenic purpura (TTP). As thrombotic microangiopathy is a medical emergency and there are more than 59 drugs known to cause TTP, we want to add alcohol and cannabis products as a secondary cause of TTP. The purpose of this study is to draw clinician's thoughtfulness regarding this deadly complication as early recognition and intensive treatment can be lifesaving.

\section{AUTHORS' CONTRIBUTION}

UR: Concept and design, data acquisition, interpretation, drafting, final approval, and agree to be accountable for all aspects of the work. RN, ZF, SA: Data acquisition, interpretation, drafting, final approval and agree to be accountable for all aspects of the work. 
Conflict of interest: Authors declared no conflict of interest.

\section{REFERENCES}

1. Stanley M, Killeen RB, Michalski JM. Thrombotic Thrombocytopenic Purpura. 2021 Sep 25. In: StatPearls [Internet]. Treasure Island (FL): StatPearls Publishing; 2021.

2. Nesheiwat Z, Goyal A, Jagtap M. Atrial Fibrillation. 2021 Aug 11. In: StatPearls [Internet]. Treasure Island (FL): StatPearls Publishing; 2021.

3. Mir MU, Khan I, Ahmed B, Abdul Razzak J. Alcohol and marijuana use while driving--an unexpected crash risk in Pakistani commercial drivers: a cross-sectional survey. BMC Public Health. 2012;12:145.

4. George JN. Clinical practice. Thrombotic thrombocytopenic purpura. N Engl J Med. 2006;354(18):1927-35.

Address for Correspondence:

Dr. Salma Salman, Dow University of Health Sciences, Karachi, Pakistan.

Email: salma.salman@duhs.edu.pk
5. Joly BS, Coppo P, Veyradier A. Thrombotic thrombocytopenic purpura. Blood. 2017;129(21):2836-46.

6. Ferrari S, Mudde GC, Rieger M, Veyradier A, Kremer Hovinga JA, Scheiflinger F. IgG subclass distribution of anti-ADAMTS13 antibodies in patients with acquired thrombotic thrombocytopenic purpura. J Thromb Haemost. 2009;7(10):1703-10.

7. Karass M, Chugh S, Andries G, Mamorska-Dyga A, Nelson JC Chander PN. Thrombotic microangiopathy associated with synthetic cannabinoid receptor agonists. Stem Cell Investig. 2017;4:43.

8. Coppo P, Veyradier A. Current management and therapeutical perspectives in thrombotic thrombocytopenic purpura. Presse Med. 2012;41(3 Pt 2):e163-76.

9. Duffy SM, Coyle TE. Platelet transfusions and bleeding complications associated with plasma exchange catheter placement in patients with presumed thrombotic thrombocytopenic purpura. J Clin Apher. 2013;28(5):356-8. 